\title{
TOUCHING base
}

Frog genetics jumping forward

Amphibians in general, and the African tree frog Xenopus laevis in particular, have taught developmental biologists a great deal. Their main limitation has been that they are not amenable to genetic manipulation. Up until a few years ago, that is, when Kirsten Kroll and Enrique Amaya described a simple and efficient method to generate transgenic frogs by injecting oocytes with nuclei from sperm heads that had been soaked in a solution containing DNA (Development 122, 3173-3183; 1996) A recent study by Nicholas MarshArmstrong and colleagues (Proc. Natl Acad. Sci. USA 96, 14389-14393; 1999) provides proof that transgenes can be passed through the germ line and stable transgenic lines established. Amaya and colleagues have obtained similar results, and developed vectors that can 'trap' endogenous genes and thereby reveal their expression patterns (Curr. Biol. 9, 1195-1198; 1999). A serious handicap for any lossof-function approach, however, is that $X$. laevis is essentially tetraploid. Luckily, there is one species of the Xenopus genus that is diploid: $X$. tropicalis has a relatively small genome (approximately the same as zebrafish and half the size of mouse) and a generation time that, at 5-6 months, is much shorter than that of $X$. laevis. $X$. tropicalis, the smaller of the two frogs shown, has all the advantages of its larger cousin, including the ability to form transgenic lines (R. Grainger, pers. comm.), and is bound to be the frog of choice for insertional mutagenesis screens and the development of targeted loss-of-function strategies.

\section{Blue Gene}

IBM's supercomputer, Deep Blue, defeated world chess champion Gary Kasparov in an epic battle in 1997. But the next generation of supercomputer from IBM-christened "Blue Gene" - will exert its considerable computational power in the service of biological research rather than in competition with the human mind. Blue Gene, due to be completed in the next five years, will be more powerful than Deep Blue by three orders of magnitude and will have an unprecedented petaflop (a million billion floating point operations per second) of computing power. It will achieve this by integrating more than one million processors, and should be two million times more powerful than the current model of computer sitting on the average desktop. First item on the agenda for Blue Gene is to model protein folding, and application of its computing power to other difficult mathematical problems of biological research-such as the inheritance of complex traits - is expected to follow quickly.

\section{"Statistics are like bikinis.}

What they reveal is suggestive, but what they conceal is vital."

\section{-Aaron Levenstein}

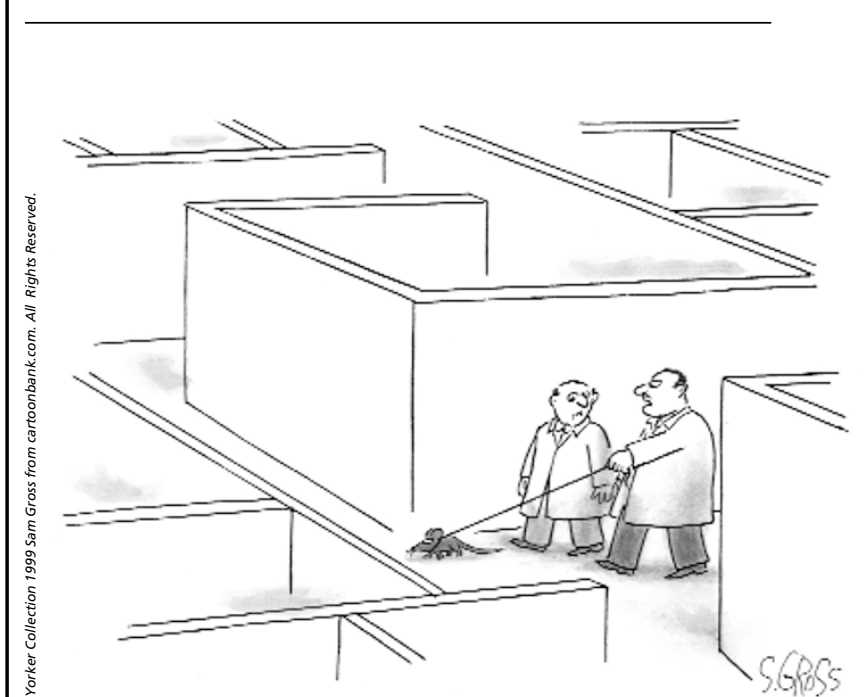

"Genetic engineering got us into this mess, and genetic engineering will get us out of it."

\section{Brain plaques in 3D}

Plaques can be thought of as blobs of 'cellular garbage'; these are routinely detected in sectioned brains of patients who have died as a consequence of Alzheimer disease. As reported recently in the Proceedings of the National Academy of Sciences (vol. 96, 14079-14084; 1999), a team led by Helene Benveniste (Duke University) has now obtained three-dimensional images of plaques in situ. A combination of high-resolution magnetic resonance microscopy and powerful computers allowed Benveniste and colleagues to conclude that "when reconstituted in a 3D image, plaque looks like small round balls, basically, spots of garbage, floating in space." For this study, the researchers used biopsies from recently deceased patients. They hope, however, that the technology will eventually allow the monitoring of plaques in vivo. Although current clinical

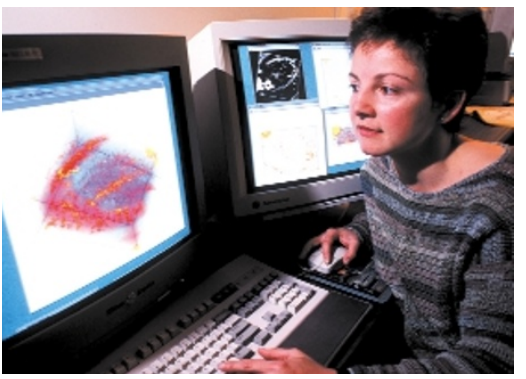
resonance technology does not have the resolution necessary to procure a detailed topography of the human brain, to obtain such images of the smaller brains of animals (such as mice) should be possible. Benveniste and colleagues have started to generate brain images of living mice, and hope to realize "the dream of every brain researcher: to follow, over time, both development of brain disease and the effects of drugs designed to combat them." 